\title{
Investigating The Contribution of Physiotherapy in The Quality of Life of Parents with Children and Adolescents suffering from Cerebral Palsy
}

\author{
Anna-Evgenia Chasidou ${ }^{1}$, Alexandra Hristara-Papadopoulou ${ }^{2 *}$, Savvas-Alexandros Zorzos ${ }^{3}$, Anna Chalkia ${ }^{4}$ \\ and Emmanouil Trevlakis ${ }^{5}$
}

${ }^{1,3}$ Physiotherapist, Alexander Technological Educational Institute of Thessaloniki, Greece

${ }^{2}$ Physiotherapist, Associate professor of physiotherapy department at ATEITh, Greece

${ }^{4,5}$ Physiotherapist, Scientific associate of physiotherapy department at ATEITh, Greece

${ }^{1,5}$ Department of physiotherapy, Alexander Technological Educational Institute of Thessaloniki,Greece

Received: July 12, 2017; Published: July 19, 2017

*Corresponding author: Alexandra Hristara-Papadopoulou, Department of ATEITh, Associate professor of physiotherapy, Department of physiotherapy, Alexander Technological Educational Institute 21 MikrasAsias St, Kalamaria-Thessaloniki, 55132, Greece,Tel: +30 2310 445879/+30 6942222445, Email: alekpap@phys.teithe.gr

Abstract

Introduction: Many researchers have focused on the quality of life (QoL) of children with cerebral palsy, but few have been done about the quality of life of parents with children diagnosed with cerebral palsy. In addition, less attention has been paid to the contribution of physiotherapy to the quality of life of parents with those children. The primary objective of this study was to evaluate the quality of life of parents with children and adolescents diagnosed with cerebral palsy. A secondary aim was to investigate the contribution of physiotherapy to the everyday life of these parents, namely that the effects of physiotherapy on the child automatically affect the quality of life of the parents.

Methods: Questionnaires were distributed to parents $(\mathrm{N}=30)$ at the Developmental Pediatrics Center "Apostolos Fokas", which belongs to the 1st Pediatric Clinic of the General Hospital of Thessaloniki "Hippocratio", as well as to a private pediatric physiotherapy center in the Thessaloniki area. The study's measurement instrument was the specialized quality of life questionnaire for parents with children and adolescents diagnosed with cerebral palsy, which designed by the first author of this dissertation and includes 3 sections (totally, 32 questions).

Results: The results showed that parents have been affected by their child's disability in different aspects of their everyday lives, such as social and family relationships, economical status and individual well-being. On the other hand, parents are involved in the intervention process and consider that physiotherapy contributes positively to the quality of life by enhancing their child's functionality.

Conclusion: According to the research hypothesis, the condition of the children, directly, affects the quality of life of parents. However, through the physiotherapeutic approach and, therefore, the functional independence of the child, the quality of life of these parents may improves.

Keywords: Cerebral Palsy; Parents; Children; Adolescents; Quality Of Life; Physiotherapy

\section{Introduction}

Cerebral palsy is one of the most common developmental disabilities that begins in early childhood and remains throughout life [1]. A child with cerebral palsy requires multiple and specialized care, which exceeds that of a normal coeval child and therefore requires more attention by parents [1]. This chronic care turns into a chronic weight, with the result that parents are often bound for a lifetime [2]. As Seligman and Darling [3] report, for some families care takes 24 hours a day, 7 days a week and for many years. All of these contribute, causing the family to suffer physically and psychologically.
Most researches have focused on the effects of the care for a child with cerebral palsy on parental mental health, but less attention has been paid to their quality of life [4-9]. Within the field of the quality of life, theWorld Health Organization (WHO) includes: physical health, mental state, independence, social relations, environment, religion, beliefs, determination and conflicting views.

According to the existing literature, the quality of life about parents with children diagnosed with cerebral palsy is lower than the quality of life of the general population and parents with normal children [4-9]. Studies suggest that mothers burden themselves 
greater with the child care [3]. In particular, parents (more so mothers) are in many cases characterized as socially isolated and limited because of the demands of the child care. Assistants in the chronic care of a disabled child (with less frequent help) appear fathers and sisters. Very little help seems to be given by relatives and friends [2].

Regarding the rehabilitation of children with cerebral palsy, it must be complete [10]. Physiotherapy plays a central role in managing a child with cerebral palsy and focuses on the functionality, active motion and optimal use of the child's potentials [10]. The goals of rehabilitation in children with cerebral palsy are to minimize the effects of physical impairments, to help the child gain independence in society and to improve the quality of life of these children and their families, who play an important role in the whole process of rehabilitation [11]. Previously, physical and occupational therapists used to focus on impairments and limitations of the child [12]. However, the provision of services in pediatric rehabilitation has undergone significant changes in recent decades [13]. The most extensive change that has taken place was the review of the role of the family. In about half a century, the role of parents in the care process has evolved into being a target for intervention.

Recent literature on pediatric rehabilitation has shown that interventions focusing on children and their families can lead to satisfaction by the care, the well-being of parents and the reduction of parental anxiety [14]. Families involved in the care of their child have the opportunity to learn more about their child and their child's treatment options. Family members, in turn, are able to share their knowledge with professionals and this provides a holistic picture of the child and, also, is a way to raise their child's awareness, as part of the family [13].

Taking into account the deficits of existing literature, the purpose of the present study was to investigate the contribution of physiotherapy to the quality of life of parents with children and adolescents diagnosed with cerebral palsy. It is widespread that the aim of physiotherapy in children and adolescents with cerebral palsy is to improve functionality, by enhancing the gross motor function and learning functional activities. Therefore, through the specially formulated quality of life questionnaire for parents with children and adolescents suffering from cerebral palsy, we assume that the functionality of the person with cerebral palsy is inextricably linked to the quality of life of their parents and guardians. Thus, if we thoroughly record the quality of life of this category of parents, we will contribute to a more holistic and sufficient physiotherapeutic intervention.

\section{Methods}

\section{Participants}

In the present study, 30 parents (20 mothers and 10 fathers) of children and adolescents (aged 3-17 years) diagnosed with cerebral palsy took part. The selection criteria pertain to parents of children and adolescents diagnosed with any form of cerebral palsy and any classification (I-V) at the Gross Motor Function Classification System (GMFCS) and age range 3-17 years. The exclusion criteria refer to mentally handicapped parents, inability to read and write the Greek language and, generally, the inability to complete this specific questionnaire. In addition, a customized written acquiescence and briefing, concerning the participation of the parents in the present dissertation, is provided, as well as their ability to be informed about the results of this study. Furthermore, the Research Ethics Committee at the Technological Educational Institute of Thessaloniki approved the study protocol.

\section{Study design}

Questionnaires were distributed to parents at the Developmental Pediatrics Center "Apostolos Fokas", which belongs to the $1^{\text {st }}$ Pediatric Clinic of the General Hospital of Thessaloniki "Hippocratio", as well as to a private pediatric physiotherapy center in the Thessaloniki area. Parents filled in the questionnaires following their oral and written briefing.

\section{Assessment of QOL}

The study's measurement instrument was the specialized quality of life questionnaire for parents with children and adolescents diagnosed with cerebral palsy, which designed and made by the first author of this dissertation and includes 3 sections (totally, 32 questions):

A. $\mathbf{1}^{\text {st }}$ section: Demographic information that includes each child's data, such as age and disability, as well as parent's data, such as occupation, educational level, living area and family structure.

B. $2^{\text {nd }}$ section: General quality of life questions from the parent questionnaire for children with disabilities (Fyka, 2006). This section includes general questions concerning parents' feelings during diagnosis, condition of the family at present, and how much disability has affected the routine of parents, and, finally, parents' opinion about physiotherapists.

C. $3^{\text {rd }}$ section: Specific questions concerning parent's opinion about physiotherapy and how it helps to improve the child's functionality and, thus, to improve the quality of life of parents.

\section{Statistical Analysis}

Data was analyzed using descriptive statistics of PSPP version 0.8.5 and Microsoft Office Excel 2010 programmes. We did not proceed to inferential statistics (t-test) due to the limited sample size $(\mathrm{N}=30)$ and the existence of qualitative variables.

\section{Results}

\section{Parents' feelings during diagnosis}

The feelings that the question included was anger, disappointment, lamentation, loneliness and shock. As for the feeling of anger, the majority of parents, in particular, the $50 \%$ answered "enough", for the feeling of disappointment the $60 \%$ of parents answered "enough", for the feeling of lamentation the $36.67 \%$ of parents answered "enough", for the feeling of loneliness the $50 \%$ answered "moderately" and for the sense of shock the $90 \%$ of parents answered "enough". The emotion of shock is the first stage that parents experience, when a child with disabilities is bornand is manifested by emotional disorganization and confusion [2]. 


\section{Time that parents spend in activities of everyday life}

Of all parents, the $43.33 \%$ spends "not at all" time on themselves, the $43.33 \%$ "not enough" and the $13.33 \%$ devotes "enough" time to themselves (Figure 1). For the time about the relationship with the partner, as a couple, the $70 \%$ of the parents responded "not enough". For the time with the family the $50 \%$ of the parents responded "not enough" and the remaining 50\% "enough". For the child's rehabilitation activities, the $80 \%$ of parents dedicate "enough" time. In addition, the $46.67 \%$ of parents dedicate "enough" time to play with their child. As for the time about friends, the $63.33 \%$ of parents responded "not enough". Finally, the $50 \%$ of parents devotes "not at all" time to their entertainment.

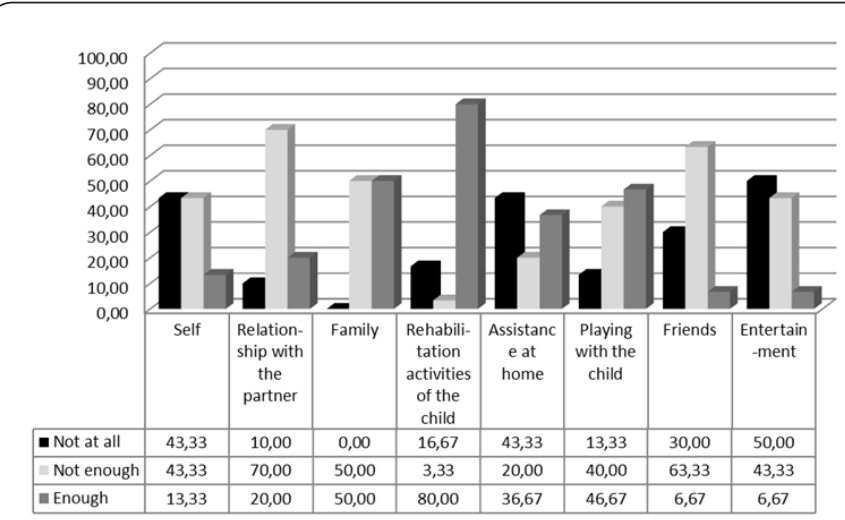

Figure 1: Time devoted by parents to people and activities of everyday life.

The influence of disability on different aspects of parental life

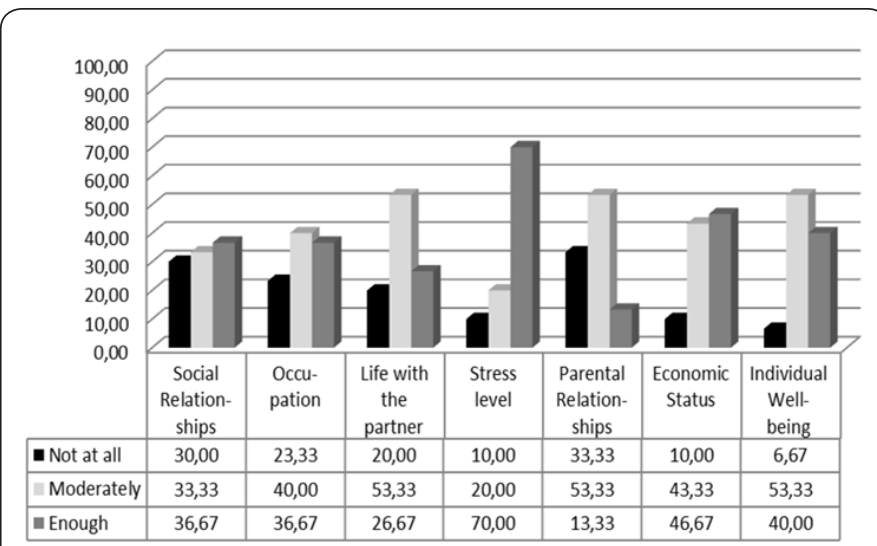

Figure 2: The influence of disability on different aspects of parental life.

This question related to the degree of which disability affects social relationships, occupation, life with the partner as a couple, level of stress, family relationships, economic status and individual welfare of parents (Figure 2). For the social relationships, the $36.67 \%$ of parents responded that the disability of the child has affected them "enough". As for the occupation, the $40 \%$ of parents has been affected "moderately" and for the life with the partner as a couple, the $53.33 \%$ of parents responded "moderately". Regarding stress level, the $70 \%$ of parents responded "enough". As for family relationships, disability has affected $53.33 \%$ of parents "moderately", while $46.67 \%$ of the parents have been affected "enough". Finally, with regard to individual well-being, the $53.33 \%$ of parents have been affected "moderately".

\section{Characteristics attributed by physiotherapists to parents}

In this question, the $53.33 \%$ of parents answered "enough" about the efficiency of physiotherapists, the $73.33 \%$ responded "moderately" to the protection afforded by physiotherapists, while for the physiotherapists' ability about care the $60 \%$ of the parents responded "moderately". In addition, for the question, in which, physio-therapists attribute stress to parents, the $53.33 \%$ responded "moderately", for anguish, the $50 \%$ of parents responded "moderately", and, finally, for stiffness, the $66.67 \%$ answered "not at all".

How qualified parents consider physiotherapists are about pathology, comprehension of family needs, prevention of stress and communicating skills

Regarding pathology, the $56.67 \%$ of parents responded that physiotherapist are "enough" qualified. In respect to comprehension of the family needs, the $56.67 \%$ responded that physiotherapist are "moderate" qualified. In terms of stress prevention, the $60 \%$ of parents responded "moderately". Finally, in regard of communication skills, the $56.67 \%$ of parents responded that they are "moderate" qualified.

\section{Age of child's physiotherapy initiation}

The majority of parents, namely $30 \%$, replied that their child started physiotherapy at the age of two months and all parents responded that they started physiotherapy immediately after the referral (Figure 3).

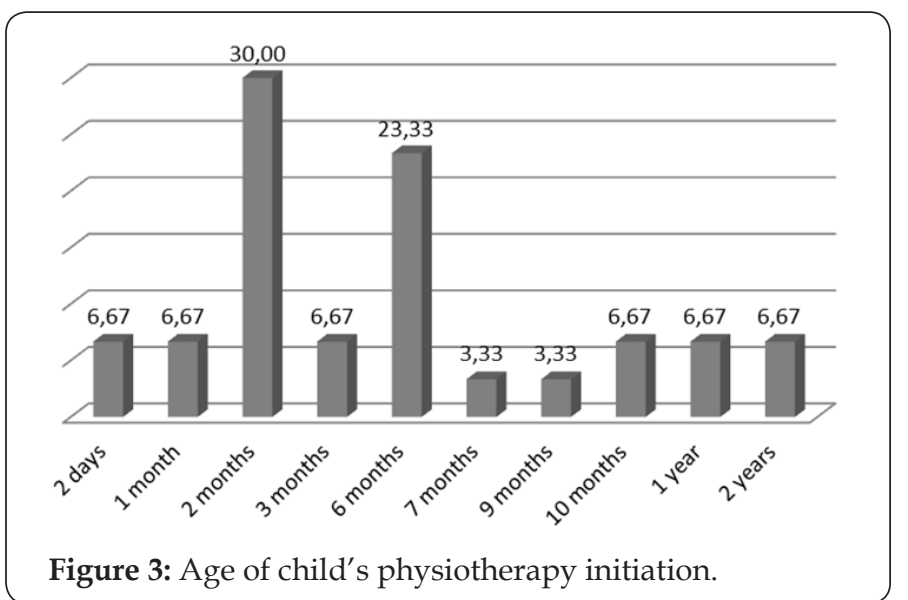

Respecting the Parent's involvement during the physiotherapeutic session: Of the total population of parents, the majority, namely $57 \%$, does not participate in the physiotherapy session, while the $43 \%$ participates. Parents who like the process of attending the physiotherapeutic session, the $60 \%$ enjoys it, while the $40 \%$ does not like it. Preference for parents to take advantage of the physiotherapeutic session's time with something else, The $67 \%$ of parents do not want to take advantage of the physiotherapeutic session's time, with something else. 
As for the encouragement by the physiotherapist about the parent's active participation in the physiotherapeutic session: According to the $70 \%$ of parents' population, the physiotherapist encourages their active participation in the session, while only the $30 \%$ says, that the physiotherapist does not encourage their participation.

\section{Opinion of the parents}

A. On the physiotherapy and the functional development of the child,

\section{B. On further occupation at home, and}

C. On the combination of physiotherapy with an alternative therapy (e.g., hippotherapy, hydrotherapy).

The $90 \%$ of parents believe that only physiotherapy is not enough for the child's functional development, and all parents believe that they need further occupation at home. Finally, in regard to the question of whether physiotherapy should be combined with alternative treatment, $80 \%$ of parents responded "yes".

In respect to the application, by parents, at home, of specialized exercises, position changes, training of activities, under the instructions of the physiotherapist, and how many hours they apply them per week, the $87 \%$ of them responded "yes". The $36.67 \%$ of them applies the specialized exercises, " 4 hours per week", the $26.67 \%$, "7 hours per week", and the $23.33 \%$, "6-7 hours per week". Also, respecting the parent or relative who applies physiotherapy at home, prescribed by the physiotherapist, the $63.33 \%$ answered that only "mother" is concerned with physiotherapy, at home, prescribed by the physiotherapist, the $33.33 \%$ answered "both parents", while only the $3.33 \%$ replied "no".

In the matter of the parents' opinion on physiotherapy and if it affects their free time, the $67 \%$ of them believes that physiotherapy affects, "enough", positively, their free time, the $23 \%$ responded "moderately", while only the $10 \%$ reported "not at all". Additionally, about the contribution of physiotherapy to the improvement of child's functionality, the $97 \%$ of parental population believes, that physiotherapy helps "enough" in the improvement of their child's functionality.

According to parents' opinion, how physiotherapy, by improving child's functionality, facilitates, by extension, their daily routine

The $97 \%$ of parental population believe that physiotherapy helps "enough" in the improvement of their child's functionality, while the $3 \%$ believes that it helps "moderately". According to parents' opinion, how physiotherapy, by improving child's functionality, facilitates, by extension, their daily routine, The $97 \%$ of parents believes that physiotherapy helps to improve child's functionality "enough" and, thus, makes easier their daily routine, while only the $3 \%$ of parents believes that physiotherapy facilitates their daily routine "moderately", by improving their child's functionality. Discussion of the parent with physiotherapists, on what concerns them, in respect to their child's progression. The $63 \%$ of parents responded that they discuss "enough" with their physiotherapist, on what it concerns them, in regard to their child's progression.

\section{Discussion}

The primary objective of this study was to investigate the quality of life of parents with children, aged 3-17 years who have been diagnosed with cerebral palsy. A secondary objective was to investigate the contribution of physiotherapy to the quality of life of these parents, specifically whether physiotherapy, by enhancing the child's functionality, also improves the quality of life of parents, as well as the parents' opinion on physiotherapy and physiotherapists and, finally, whether the last ones support the model of familycentered therapeutic approach.

In terms of parents' quality of life, questions were asked about the condition of the family at present, namely the time that parents spend on people and activities of everyday life, and how disability affects different aspects of their everyday lives. From the results it turned out that parents do not spend enough time on themselves or even on the relationship with their partner, as a couple. As for their family, half of the parents answered that they spend enough time, while the remaining half do not spend enough. In regard of child's rehabilitation activities, the majority of parents spend enough time. The majority of them, also, spend enough time, playing with their child. Finally, most parents do not spend enough time with their friends and the majority of parents do not spend any time at all about their entertainment.

In addition, the child's disability seems to affect the daily routine of the parents. In particular, it greatly affects parents' social relationships, stress levels and economic conditions, while moderately affects occupation, life with the partner, family relationships and individual welfare of parents. As confirmed by literature, the solicitude for a child with cerebral palsy may positively affect a parent's ability to create new social support networks, but the condition of such a child's care may adversely affect parents' physical health, social relationships, freedom and independence, family relationships and economic stability [14].

From the knowledge, regarding parents' opinion about physiotherapists, it seems that the last ones are highly competent, in terms of efficiency, they attribute moderate protection and ability, in terms of solicitude, to parents, little anxiety and anguish, while they do not express any stiffness at all. Also, according to parents' opinion, physiotherapists are highly trained in pathology, while they are themselves moderately satisfied with regard to the comprehension of family needs, the prevention of stress, as well as communicating skills. However, most parents discuss with their physiotherapists what they are concerned about their child's development, and they trust their opinion and advice. The role of parents in the health care process and the attitudes of healthcare professionals, are essential elements in understanding care processes [12].

With the family unit as the primary framework for the promotion of children's health and well-being, the family-centered approach has been shown to contribute to the emotional wellbeing of parents [15-17]. For this reason, in the questionnaire of this dissertation questions about the involvement of parents in physiotherapeutic sessions included. The results also showed 
that the majority of parents did not participate during the physiotherapy session. However, parents who participate in the physiotherapy session often enjoy the process of attendance, while very few of them would prefer to use this time with something else. According to the opinion of parents, the majority of themselves do not participate in the physiotherapy session, although the physiotherapist encourages their active participation.

Parents' beliefs, early on, in terms of their child's treatment, may have a significant impact on the attendance during therapeutic session and, consequently, on the therapeutic outcomes [18]. In addition, parents have been shown to be more satisfied with family-centered interventions [15]. Regarding the question about physiotherapy and whether suffices for the functional development of the child, the majority of parents believes that is not sufficient in itself, whereas all parents believe that further work at the home with physiotherapeutic exercises is needed. Most of the parents, also, believe that physiotherapy should be combined with an alternative therapy. Most parents implement specialized exercises, position changes and activities training, under the guidance of the physiotherapist, at home. However, the majority of parents spends less than four hours per week, proving either that they do not have enough time, due to daily duties, or because of the psychological and physical weariness they experience both of the parents.

Finally, with regard to the contribution of physiotherapy to the quality of life of parents, it seems, initially, that physiotherapy, according to the parents' opinion, helps to improve the child's functionality. In addition, by improving child's functionality, the majority of parents believes that it facilitates, as a result, their daily routine. Many of the parents, also, believe that physiotherapy, positively, affects their free time. However, in this dissertation, there were some limitations. Initially, the sample was limited $(\mathrm{n}=$ 30), as many parents refused to complete the questionnaire. After that, the questionnaire did not cover all the dimensions of quality of life. In the sequel, the study consisted of children with a large age range (3-17 years), which affects the children's demands in terms of their parents care and, hence, their quality of life. Therefore, studies with a more specific age range need to be conducted. The study, also, included all forms of cerebral palsy and all levels of GMFCS (Gross Motor Function Classification System), which may again affect the quality of life of parents. Finally, research bibliography at the databases (scopus.com; sciencedirect.com; pubmed.com), which concerned with the quality of life of parents with children and adolescents diagnosed with cerebral palsy, was deficient.

\section{Conclusion}

According to the results of the present study, which are perfectly consistent with the initial hypothesis, physiotherapy seems to contribute positively to the quality of life of parents with children and adolescents suffering from cerebral palsy. This is achieved by increasing the child's functionality, involving parents in the intervention process, and implementing family-centered therapeutic approach, where parents learn their child better and their care is facilitated. However, further researches, in regard to the contribution of physiotherapy to the quality of life of parents, with a larger sample of subjects, needs to be carried out.

\section{References}

1. Guillamon Noemi, Ruben Nieto, Modesta Pousada, Diego Redolar, Elena Munoz, et al. (2012) Quality of life and mental health among parents of children with cerebral palsy: the influence of self-efficacy and coping strategies. Journal of Clinical Nursing 22(11): 1579-1590.

2. Tsimpidaki A (2013) Child with special needs, family and school. A relationship in interaction. 73-84.

3. Seligman M, Darling B (2007) Ordinary families, special children. 3rd edn. New York: The Guilford Press.

4. Davis E, Shelly A, Waters E, Boyd R, Cook K, et al. (2010) The impact of caring for a child with cerebralpalsy: quality of life for mothers and fathers. Child: Care, Health and Development 36: 63-73.

5. Kaya K, Unsal-Delialioglu S, KutayOrdu-Gokkaya N, Ozisler Z, Ergun $\mathrm{N}$, et al. (2010) Musculoskeletal pain, quality of life and depression in mothers of children with cerebralpalsy. Disability and Rehabilitation 32(20): 1666-1672.

6. Prudente COM, Barbosa MA, Porto CC (2010) Relation between quality of life of mothers of children with cerebralpalsy and the children's motor functioning, after ten months of rehabilitation. Revista LatinoAmericana de Enfermagem 18(2): 149-155.

7. Romeo DM, Cioni M, Distefano A, Battaglia LR, Costanzo L, et al. (2010) Quality of life in parents of children with cerebralpalsy: is it influenced by the child's behaviour?. Neuropediatrics 41(3): 121-126.

8. Okurowska-Zawada B, Kulak W, Wojtkowsku J, Sienkiewicz D, PaszkoPatej G (2011) Quality of life of parents of children with cerebral palsy. Progress in Health Sciences 1: 116-123.

9. Ström H, Kreuter M, Rosberg S (2011) Quality of life in parents/ caretakers of children with cerebral palsy in Kampong Cham, Cambodia. Journal of Tropical Pediatrics. 58(4): 303-306.

10. Mintaze Kerem Gunel (2009) Rehabilitation of children with cerebral palsy from a physiotherapist's perspective. Acta Orthop Traumatol Turc 43(2): 173-180.

11. Cusick A, McIntyre S, Novak I, Lannin N, Lowe K (2006) A comparison of goal attainment scaling and the Canadian Occupational Performance Measure for paediatric rehabilitation research. Pediatric Rehabilitation. 9(2): 149-157.

12. Bly L (1991) A historical view and current view of the basis of NDT. Pediatric Physical Therapy 3: 131-135.

13. Siebes R, L Wijnroks, M Ketelaar, PEM van Schie, JW Gorter (2006) Parent participation in paediatric rehabilitation treatment centres in the Netherlands: a parents' viewpoint. Blackwell Publishing Ltd, Child: care, health and development. 33(2): 196-205.

14. Alsem M, M Verhoef, JW Gorter, LCM Langezaal, JMA Visser-Meily, M Ketelaar (2016) Parents' perceptions of the services provided to children with cerebral palsy in the transition from preschool rehabilitation to school-based services. John Wiley \& Sons Ltd Child: care, health and development 42(4): 455-463.

15. King G, Rosenbaum P, King S (1996) Parents' perceptions of care giving: development and validation of a measure of processes. Developmental Medicine and Child Neurology 38(9): 757-772.

16. Rosenbaum P, King S, Law M, King G, Evans J (1998) Family-centred service: a conceptual framework and research Overview. Physical and Occupational Therapy in Pediatrics. 18(1): 1-20.

17. Raina P, O’Donnell M, Rosenbaum P, Brehaut J, Walter SD, et al. (2005) The health and well-being of caregivers of children with cerebral palsy. Pediatrics 115: 626-636.

18. Nock MK, Ferriter C, Holmberg E (2007) Parent beliefs about treatment credibility and effectiveness: assessment and relation to subsequent treatment participation. Journal of Child and Family Studies 16: 27-38. 


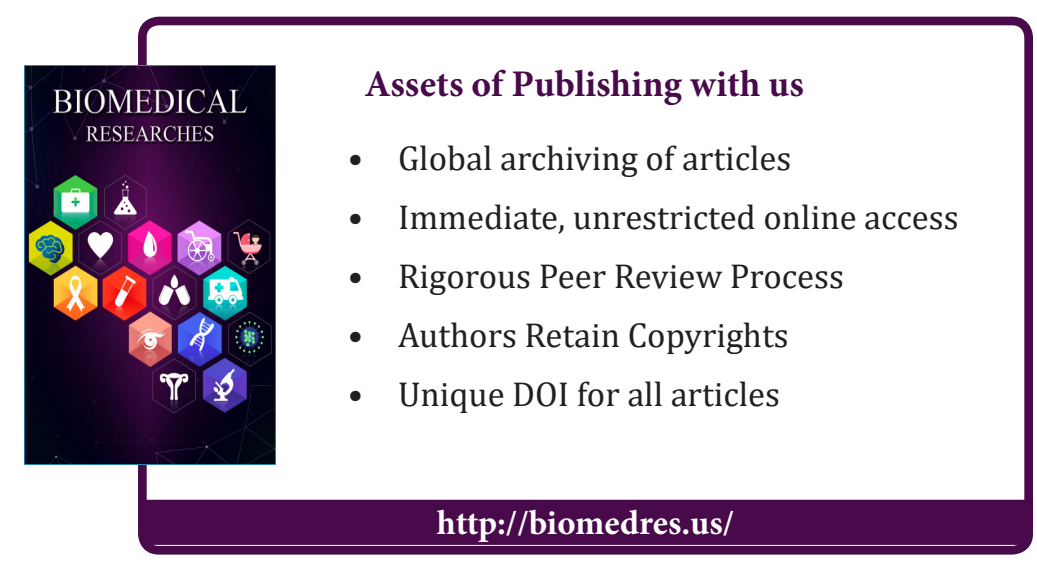

